\title{
Método para calcular el valor agregado en cadenas de suministro de productos electromecánicos
}

\author{
Method to calculate the added value in supply chain of \\ electromechanical products \\ Andrey Vinajera-Zamora ${ }^{*} \quad$ Fernando Marrero-Delgado $^{1} \quad$ Mariana Ruiz-Morales $^{2}$ \\ Recibido 18 de mayo de 2016, aceptado 24 de agosto de 2016 \\ Received: May 18, 2016 Accepted: August 24, 2016
}

\begin{abstract}
RESUMEN
En el presente artículo se propone un método para calcular el valor agregado en las operaciones de la cadena de suministro de productos electromecánicos compuesta por los procesos de aprovisionamiento, producción y distribución. El objeto de estudio específico lo constituye la cadena de suministro de contadores de energía eléctrica. La herramienta propuesta está compuesta por varios pasos relacionados con la definición del precio de venta del producto, la identificación de las operaciones de los procesos y de los requerimientos operacionales, el cálculo del coeficiente de valor agregado y del valor de cada producto en cada operación, y el cálculo de la cantidad de recursos financieros requeridos para comprar la materia prima. Se obtuvo como resultado que la operación de mayor valor agregado neto por unidad es la de calibración de contadores de energía eléctrica mientras que la de mayor valor agregado es la de almacenaje de productos terminados. Las principales conclusiones están asociadas a la forma de analizar y calcular el valor agregado en cadenas de suministros compuestas por los procesos de aprovisionamiento, producción y distribución o algunos de estos, tomando como base las deficiencias detectadas en otros métodos donde se calcula el valor agregado.
\end{abstract}

Palabras clave: Cadena de valor, valor económico agregado, gestión de la cadena de suministro.

\begin{abstract}
This article proposes a methodology for calculating the added value in the operations of the supply chain of electromechanical products formed by the processes of procurement, production and distribution. The object of study constitutes the supply chain of electrical energy meters. The proposed tool consists of several steps related to the definition of the selling price of the product, the identification of operations of each process and operational requirements, calculating the coefficient added value and value of each product in each operation, and calculating the amount of financial resources required to buy the raw material. It was obtained as a result that the operation of higher net added value per unit is the calibration of electrical energy meters while the higher added value is the storage of finished products. The main findings are associated with how to analyze and calculate the value-added supply chains composed of procurement processes, production and distribution or some of these, based on the deficiencies identified in other methods where the added value is calculated.
\end{abstract}

Keywords: Value chain, economic value added, supply chain management.

1 Departamento de Ingeniería Industrial. Universidad Central "Marta Abreu” de Las Villas, Carretera a Camajuaní Km 5 ํㅗ․ Santa Clara, Cuba. E-mail: avinajera2011@gmail.com; fmarrero@uclv.edu.cu

2 Departamento de Ingenierías. Universidad Iberoamericana de México. Prolongación Paseo de Reforma 880. Ciudad de México, D.F., México. E-mail: mariana.ruiz@ ibero.mx

* Autor de correspondencia 


\section{INTRODUCCIÓN}

La cadena de suministro como sistema de empresas para transformar materia prima en bienes intermedios, luego en productos terminados, y finalmente entregarlos a los clientes [1-3] debe ser coordinada y optimizada con el objetivo de lograr un alto nivel de servicio al cliente [4] mediantes diferentes procesos y actividades que generen valor para los clientes finales en forma de producto o servicio [5]. A su vez, estos procesos y operaciones generan un costo de producción el que varía de acuerdo al volumen de producción y la variedad en el surtido. Dichos costos, llevados a términos generales (totales) están relacionados con la cadena de valor y con la rentabilidad máxima obtenida de la mayor producción en esta [6].

La cadena de valor, la que es considerada un conjunto de actividades relacionadas de valor agregado [7], es capaz de identificar las actividades, funciones y procesos de negocios que deben ser realizados en el diseño, en la producción, en el mercado, en la entrega y en el apoyo al producto y/o servicio [8]. No obstante, la colaboración entre las organizaciones que componen la cadenas de valor es considerada la clave para una mejor gestión de la cadena de suministro y, por ende, un mejor funcionamiento [9].

De esta forma, para lograr un mejor análisis de la cadena de valor económica como herramienta útil que brinda información para la coordinación y optimización de las actividades [10], se hace imprescindible el cálculo del valor agregado en cada uno de las operaciones que componen la cadena de suministro [11], incluso, puede resultar de vital importancia en las cadenas donde el objetivo no es económico, como las cadenas de suministros sociales [12]. Sin embargo, el valor económico agregado (Economic Value Added, EVA) juega un papel muy importante en la cadena de suministro, ya que según los accionistas, como medida de rendimiento es considerado el mejor indicador para evaluar la creación de valor en esta [13].

Por otra parte, aunque el propósito financiero básico de una empresa es la maximización de su valor, las estrategias para lograr este objetivo se ejecutan con un enfoque en el riesgo y la incertidumbre [14]. Aun así, este valor puede ser maximizado si se adaptan los enfoques existentes de la cadena de valor a enfoques con perspectivas sustentables [15].

Las técnicas modernas de evaluación basadas en teorías económicas como: EVA, valor económico agregado refinado (Refined Economic Value Added, REVA), valor agregado del mercado (Market Value Added, MVA), valor agregado por los accionistas (Shareholder Value Added, SVA) y el valor agregado del efectivo (Cash Value Added, CVA) han remplazado los criterios basados en datos financieros. A pesar de las numerosas aplicaciones de los instrumentos anteriormente mencionados, teóricamente no están relacionados con la cadena de valor [16]. Sin embargo, si se combina el análisis de la cadena de valor y el análisis del ciclo de vida del producto, se puede determinar cuáles son las actividades que agregan valor en cada uno de los niveles de la cadena [17].

Asimismo, en la literatura de producción en economía, el término "valor agregado" es el más usado para analizar cuando el valor de un artículo aumenta en cada estado del proceso de producción [18]. Por otro lado, aunque el valor agregado es considerado el conjunto de actividades en una empresa destinadas a la creación de un producto o servicio valorado por el cliente [8], también resulta conocido como valor agregado económico, el que ha sido adoptado en disímiles ocasiones para medir el desempeño de un proceso y puede variar según las características de la empresa [19]. Por tal motivo, y dada la similitud de los conceptos de valor agregado y valor económico agregado, se utilizará de forma indistinta estos dos términos en el presente artículo.

Por otro lado, aunque existen varios métodos para calcular el valor agregado [11, 18, 20-25] en la literatura consultada; se puede afirmar que la mayoría se enfocan en estimaciones globales, o sea, a un proceso específico o a la cadena de suministro en sí. Esto trae como consecuencia la dificultad para estimar el valor que se agrega a un bien en cada uno de las operaciones de la cadena de suministro que los produce.

En tal sentido, la presente investigación tiene como objetivo proponer un método para el cálculo del valor agregado en las operaciones pertenecientes a los procesos de aprovisionamiento, producción y distribución. Al mismo tiempo, permitirá conocer 
cuánto valor agrega cada operación ${ }^{3}$ que componen dichos procesos y cuál de estas es identificada como el cuello de botella según su valor agregado neto. Conjuntamente, el cálculo del valor inicial en la primera operación, permite saber de cuántos recursos financieros se dispone para comprar la materia prima. El caso de estudio del presente artículo será la cadena de suministro de contadores de energía eléctrica, perteneciente a la Fábrica de Fusibles y Desconectivos de Villa Clara, Cuba.

\section{ANÁLISIS TEÓRICO}

\section{Análisis teórico sobre valor agregado}

En el mundo competitivo de hoy, el valor y la creación de riqueza para los accionistas se encuentran entre los objetivos más importantes a lograr. Con el fin de alcanzar sus objetivos, el inversionista necesita algunos instrumentos para medir el valor potencial de cada oportunidad de negocio (retorno de la inversión y ganancias por acción) y está claro que estos instrumentos no son capaces de predecir exactamente el futuro pero proveen información valiosa y ayudan al inversor en el proceso de toma de decisiones [26].

Por otro lado, el mejoramiento de la cadena de valor es un objetivo clave que generalmente conlleva a mejores desempeños en términos de costo y calidad [27]. Al mismo tiempo, las operaciones de valor agregado en la cadena de suministro son vitales para el éxito de cualquier cadena [28]. Para tal fin, resulta imprescindible calcular el valor que se agrega a un producto en cada una de los procesos de la cadena de suministro por donde pasa. A su vez, [20] define el valor agregado como la diferencia en valor económico entre las entradas físicas y las salidas de un proceso de manufactura.

Como se abordó en la introducción del presente artículo, según [16], entre las técnicas existente para determinar el valor agregado se encuentran: EVA, REVA, MVA, SVA y CVA. En tal sentido, en la Tabla 1 se muestran varias investigaciones que determinan el valor agregado en cadenas de suministro.

$\mathrm{Al}$ analizar la Tabla 1, se puede observar la tendencia a calcular el valor agregado de la cadena de suministro o procesos de esta, y no a las operaciones que la componen. En tal sentido, [20] estimaron el valor agregado a cada producto analizado. Sin embargo, dicho método fue diseñado para una cadena de suministro específica (industria forestal) y calcula el valor agregado del proceso de manufactura y no de las operaciones que lo componen. Por otro lado, [23] determina el valor del producto final por medio de la suma del valor agregado bruto (Gross Added Value, GVA) y el valor de los productos intermedios.

Tabla 1. Contribuciones en el cálculo del valor agregado.

\begin{tabular}{|c|c|c|c|c|c|c|c|c|c|c|c|c|}
\hline \multirow{2}{*}{ Autor(es) } & \multicolumn{4}{|c|}{ Valor agregado que calcula } & \multicolumn{3}{|c|}{ Enfoque del cálculo } & \multicolumn{4}{|c|}{ Objeto de estudio } \\
\cline { 2 - 13 } & $\mathbf{C S}$ & $\mathbf{M}$ & $\mathbf{A}$ & $\mathbf{E f}$ & Op. & Ot. & Global & Detallado & CS & PA & PP & PD \\
\hline$[18]$ & & & & & & $\mathrm{X}$ & $\mathrm{X}$ & & & & & $\mathrm{X}$ \\
\hline$[29]$ & & & $\mathrm{X}$ & & & & $\mathrm{X}$ & & $\mathrm{X}$ & & & \\
\hline$[11]$ & & & & & $\mathrm{X}$ & & & $\mathrm{X}$ & & & $\mathrm{X}$ & \\
\hline$[23]$ & $\mathrm{X}$ & & & & & & $\mathrm{X}$ & & $\mathrm{X}$ & & & \\
\hline$[13]$ & & $\mathrm{X}$ & & & & & $\mathrm{X}$ & & & & & $\mathrm{X}$ \\
\hline$[16]$ & & & $\mathrm{X}$ & & & & $\mathrm{X}$ & & $\mathrm{X}$ & & & \\
\hline$[20]$ & $\mathrm{X}$ & & & & & & $\mathrm{X}$ & & & & $\mathrm{X}$ & \\
\hline$[30]$ & $\mathrm{X}$ & & & & & & $\mathrm{X}$ & & $\mathrm{X}$ & & & \\
\hline$[21]$ & $\mathrm{X}$ & & & & & & $\mathrm{X}$ & & $\mathrm{X}$ & & & \\
\hline$[31]$ & $\mathrm{X}$ & & & & & & $\mathrm{X}$ & & $\mathrm{X}$ & & & \\
\hline$[32]$ & & & & $\mathrm{X}$ & & & $\mathrm{X}$ & & $\mathrm{X}$ & & & \\
\hline
\end{tabular}

CS: Cadena de suministro; M: Mercado; A: Accionistas; Ef.: Efectivo; Op.: Operaciones; Ot.: Otro; El: Elemento de la cadena de suministro; PA: Proceso de aprovisionamiento; PP: Proceso de producción; PD: Proceso de distribución.

\footnotetext{
3 Se utilizará el término "operación” para reflejar indistintamente operaciones u otras actividades que conformen un proceso.
} 
Por su parte, el GVA es calculado mediante la suma del valor agregado de la tecnología, el capital y el conocimiento, y no de las operaciones que componen la cadena de suministro.

[21] determinan el EVA por medio de la diferencia entre la utilidad neta después de impuestos (NOPAT) y la inversión de la cadena de suministro. Este método a su vez, permite la evaluación de manera realista de la utilidad de operación mediante la inclusión de su propio capital invertido en el negocio después del conteo de los ingresos y los intereses [6]. Por otro lado, aunque al aplicar dicha herramienta se desconoce el valor que se agrega en cada una de las operaciones que componen la cadena de suministro, esta proporciona una medida de desempeño altamente correlacionada con la riqueza de los accionistas la que responde a las acciones de los directivos y proporciona información relevante para el proceso de toma de decisiones [13].

No obstante, aunque en la práctica contable, existen grandes variaciones en la interpretación precisa de valor económico añadido [26], la literatura reporta que las grandes compañías están decididas a adoptar las medidas de desempeño del EVA como guía principal para sus políticas corporativas [18]. Sin embargo, se puede identificar como principal debilidad de EVA que no puede ser aplicado a cada una de las operaciones que componen la cadena de suministro. Esto se debe a que al depender de NOPAT, tiene que considerar el producto y/o servicio terminado, y no una parte del mismo.

Por otro lado, [30] determinan el REVA mediante la diferencia entre NOPAT y la multiplicación del WACC y el valor total del mercado. A su vez, el indicador REVA es considerado teóricamente superior al EVA [33]. No obstante, aunque ha sido menos aplicado y depende también de las ganancias y las inversiones [34], presentando la misma debilidad que EVA. En el caso de [31], calculan el valor agregado pero enfocado a la sostenibilidad de la cadena. El objetivo de esta método es determinar si una cadena de suministro es sostenible de acuerdo a un valor de referencia escogido $^{4}$, por lo que no permite calcular el valor agregado en las operaciones de esta.

4 Este valor de referencia puede ser de otra empresa, un sector en particular, economía o incluso una meta establecida internacionalmente [39].
[13] calcula el valor agregado del mercado el que depende del valor total del mercado de la compañía y el capital invertido. Dicho indicador presenta como debilidad que, al analizar el valor agregado del mercado, depende de los bienes y/o servicios terminados que la empresa sea capaz de brindar. $\mathrm{Al}$ mismo tiempo, solo analiza el proceso de distribución de la cadena de suministro.

[29] y [16] determinan el valor agregado de los accionistas por conducto de equidad del valor del mercado, dividendos, pagos a los accionistas, inversiones y conversión de las obligaciones. [32] calcula el valor agregado del efectivo utilizando variables como utilidades después de impuestos, depreciación, débito, depreciación económica, equidad del valor en el libro y el costo medio ponderado del capital. [18] determinó el valor agregado en uno de los elementos que componen el proceso de enseñanza (profesores). Como se puede observar, estos tres valores agregados son enfocados a elementos de la cadena de suministro lo que limita su aplicación en las operaciones de esta.

Por otro lado, solo [11] calculó el valor agregado en cada una de las operaciones de la cadena de suministro, en la cual incluye ecuaciones para determinar su valor inicial y final. Sin embargo, dicho método solo puede ser aplicada en los procesos de producción, dejando al margen los procesos de aprovisionamiento y distribución de la cadena de suministro.

Una vez analizados estos métodos se puede concluir que solo el método propuesto por [11] puede ser aplicada para determinar el valor agregado de cada una de las operaciones, pero solo de procesos de producción. De esta forma, queda evidenciado que ninguna de estas investigaciones puede ser utilizada para determinar el valor agregado en las operaciones de la cadena objeto de estudio del presente artículo, sin antes realizarle algunas modificaciones.

Por tal motivo, y como base para crear un método que permita calcular el valor agregado en las operaciones de cadenas de suministro compuesta por los procesos de aprovisionamiento, producción y distribución, se tomará el de [11] ya que aunque analiza solo las operaciones de uno de los procesos, sí permite calcular el valor agregado de estas. 


\section{MÉTODO PARA CALCULAR EL VALOR AGREGADO}

Los pasos del método propuesto por [11] se muestran en en la Figura 1, los que serán tomados como guía para el cálculo del valor agregado.

1.- Definición precio de venta del producto

$$
\text { Љ }
$$

2.- Identificación de las operaciones de los procesos

马

3.- Identificación de los requerimientos operacionales

Љ

4.- Cálculo del coeficiente de valor agregado

万.

5.- Cálculo del valor de cada producto en cada operación

5

6.- Cálculo de la cantidad de recursos financieros requeridos para comprar la materia prima

Figura 1. Método para calcular el valor agregado. Fuente: [11].

Dicho método conservará su estructura original, al que se le ajustará la ecuación para el cálculo de los pesos de cada una de las operaciones, de forma tal que permita su aplicación en la cadena objeto de estudio del presente artículo. El mismo, una vez modificado tiene como principal fortaleza que puede ser aplicado a los procesos de aprovisionamiento, producción y distribución de cualquier cadena de suministro o a algún proceso específico. Los pasos del método propuesto serán descritos a continuación.

\section{Paso 1. Definición del precio de venta del producto} Según [35], el precio de venta de un producto es una decisión que depende del precio de los competidores y del costo de producción, donde el primero es considerado un punto de referencia basado en los costos y en el valor agregado, y el costo una función de los recursos empleados para obtener un producto [36]. A su vez, el precio debe ser definido según el por ciento que decida la cadena o el Estado (en caso de ser una empresa estatal) para la utilidad del producto en base a su costo de producción y, al mismo tiempo, debe estar en el rango de los precios del mismo producto vendido por los competidores.

\section{Paso 2. Identificación de las operaciones de los procesos}

Un proceso es un sistema coordinado de operaciones en el curso de las cuales el producto obtiene valor agregado [27]. Cada operación debe ser identificado de acuerdo a su capacidad, aunque esta en ocasiones no solo dependa de las capacidades de las máquinas, sino de los niveles de fuerza de trabajo disponibles [37]. Por esto, la capacidad de producción será calculada de forma diferente en cada una de las operaciones.

En el caso de los procesos de aprovisionamiento y distribución la capacidad de procesamiento se determina mediante la ecuación (1) donde las actividades efectuadas dependerán del tiempo empleado para: el transporte, la realización de solicitudes de compra o de ventas, llamadas telefónicas, correo, etc. Además, estos procesos siempre requieren autorización de la gerencia debido a que incurren en un costo adicional para la cadena.

$$
C p_{i j}=\frac{P_{i j}}{t_{i j}}
$$

Donde $C p_{i j}$ es la capacidad de procesamiento del producto $j$ en la operación $i ; P_{i j}$ es la cantidad de productos que se soliciten, se transporten o se compren del producto $j$ en la operación $i$ y $t_{i j}$ es el tiempo total empleado para el producto $j$ en la operación $i$. En el caso de las operaciones en el proceso de producción, la capacidad se calculará a través de la ecuación (2) donde $r_{j}$ es el peso específico del producto $j$ en el volumen total de producción, $F_{j}$ es el fondo de tiempo de la operación $i$ y $n$ la cantidad de productos.

$$
\operatorname{Cpr}_{i j}=r_{j} * \frac{F_{i}}{\sum_{i=j}^{n} * t_{i j}}
$$

\section{Paso 3. Identificación de los requerimientos operacionales}

De acuerdo con [38] los requerimientos operacionales pueden ser identificados mediante la lista de materiales, componentes y otros recursos necesarios para la producción del bien y/o el servicio. 
Paso 4. Cálculo del coeficiente de valor agregado En este paso se calculará el coeficiente de valor agregado en la operación $i$ para el producto $j\left(S w_{i j}\right)$ en los procesos de aprovisionamiento, producción y distribución. Para esto se hace necesario calcular el tiempo de procesamiento ${ }^{5}$, costos y tiempo de retraso $^{6}$ en cada uno de las operaciones de la cadena de suministro. Este coeficiente de valor agregado representa el porcentaje del valor agregado neto del producto $j$ que le pertenece a la operación $i$. Para el cálculo de $S w_{i j}$ y el valor agregado a cada una de las operaciones de la cadena de suministro posteriormente, se utilizará el procedimiento siguiente:

a) Caracterización de las operaciones del proceso.

En este paso deben ser identificados los recursos materiales y humanos en cada operación. Al mismo tiempo se tendrán que determinar los costos presentes (costos directos e indirectos) en cada uno de estos.

b) Cálculo del tiempo de procesamiento. El tiempo de procesamiento se calcula dependiendo del proceso de la cadena donde se encuentre la operación objeto de estudio. En el caso de los procesos de aprovisionamiento y distribución, el tiempo de procesamiento del producto $j$ en la operación $i\left(t_{i j}\right)$ depende del tiempo que demoren algunos servicios a solicitar para el producto $j$ en el operación $i\left(t s_{i j}\right)$ (incluye tiempo para realizar e imprimir documentos necesarios, autorización y firma de estos), posibles viajes que se tengan que hacer para el producto $j$ en el operación $i\left(t t_{i j}\right)$ y $t c_{i j}$ es el tiempo del producto $j$ en la operación $i$ utilizado en llamadas telefónicas, correo electrónico o para revisar algún sitio web en busca de alguna información. En caso de que algunos de estos tiempos $\left(t s_{i j}, t t_{i j}\right.$ y $\left.t c_{i j}\right)$ se realicen de forma simultánea, se tomará el de mayor valor y los tiempos restantes se tomarán como cero. En resumen, este tiempo de procesamiento se calculará de acuerdo con la ecuación (3).

$$
t_{i j}=t s_{i j}+t c_{i j}+t t_{i j}
$$

\footnotetext{
5 Se utilizará el término "tiempo de procesamiento" para el tiempo que lleve realizar una operación en cualquiera de los procesos de la cadena (aprovisionamiento, producción y distribución).

6 Se utilizará el término "tiempo de retraso" para reflejar el atraso en el aprovisionamiento, en la producción o en la distribución.
}

En el caso del tiempo de procesamiento en el proceso de manufactura, depende del tipo de operación y se calcula mediante la ecuación (4), (5) о (6).

$$
\text { Operación mecánica } \quad t_{i j}=t m_{i j}
$$

Operación mecánica manual $t_{i j}=t m_{i j}+p t_{i}$

Operación manual

$$
t_{i j}=p t_{i j}
$$

Donde $m t_{i j}$ es el tiempo utilizado por el o los equipos en la operación $i$ en el producto $j, p t_{i}$ es el tiempo de procesamiento de la operación $i$ en el producto $j$. El $p t_{i j}$ considera el tiempo de transporte hasta la operación $i$ y el tiempo de preparación de los componentes a ser procesados en dicha operación [11].

c) Cálculo del tiempo de retraso. En el caso del tiempo de retraso del producto $j$ en la operación $i\left(D t_{i j}\right)$, se calcula empleando la ecuación (7).

$$
D t_{i j}=t_{i j} \min \left(t_{i j}\right)
$$

d) Cálculo del costo: el costo a tener en cuenta en cada uno de las operaciones dependerá de cuánto se gaste mientras se procese un artículo en dicha operación. Para esto, se tendrán en cuenta los costos directos e indirectos del producto $j$ en la operación $i$ respectivamente $\left(I C_{i j}\right.$ y $\left.D C_{i j}\right)$, permitiendo calcular el costo del producto $j$ en la operación $i\left(C_{i j}\right)$ empleando la ecuación (8). En el caso de que exista un costo indirecto para varias operaciones, el $I C_{i j}$ será calculado por medio de la ecuación (9) donde $\mathrm{n}$ representa la cantidad de operaciones de la cadena de suministro.

$$
\begin{gathered}
C_{i j}=C I_{i j}+C D_{i j} \\
C_{i j}=\frac{C I_{i j}}{n}
\end{gathered}
$$

e) Cálculo del coeficiente de valor agregado: Una vez que se determina cada uno de los elementos necesarios para determinar el valor 
agregado, se procederá al cálculo del peso específico de la operación $i$ en el producto $j$ o coeficiente de valor agregado $\left(S w_{i j}\right)$ mediante de la ecuación (10).

$$
S w_{i j}=\frac{1}{3}\left(\frac{t_{i j}}{T_{j}}+\frac{D t_{i j}}{D t_{j}}+\frac{C_{i j}}{C_{j}}\right)
$$

Donde $D t_{j}$ es el tiempo atrasado en el producto $j$ (suma de todos los $D t_{i j}$ ), $T_{j}$ es el tiempo total de demora el producto $j$ (suma de todos $\operatorname{los} t_{i j}$ ) y $C j$ es el costo total del producto $j$ (suma de todos los $I C_{i j}$ y los $D C_{i j}$.

f) Cálculo del valor agregado neto: el valor agregado neto $\left(N A V_{i j}\right)$ en la operación $i$ del producto $j$ se determinará por la ecuación 11 , donde $B j$ es el ingreso neto unitario del producto $j$.

$$
N A V_{i j}=S w_{i j} * B_{j}
$$

Una vez determinado el $N A V_{i j}$, se determinará el valor que se agrega al producto $j$ en la operación $i\left(A V_{i j}\right)$ por medio la ecuación 12, donde $A c_{i j}$ es el costo agregado al producto $j$ en la operación $i$ el cual debe ser menor o igual que $C_{i j}$.

$$
A V_{i j}=N A V_{i j}+A C_{i j}
$$

\section{Paso 5. Cálculo del valor de cada producto en cada operación}

Por otra parte, el valor de cada producto en cada operación se determinará de acuerdo con la ecuación (13) donde $I V_{i j}$ es el valor de entrada del producto $j$ al proceso $i$ y $O V_{i j}$ es el valor de salida del producto $j$ en la operación $i$. Esta ecuación debe ser aplicada a las operaciones de atrás hacia delante, donde el valor final del producto coincide con el precio de venta y el valor final del producto $j$ en la operación $i$ coincide con el valor inicial del producto $j$ en la operación $i+1$.

$$
I V_{i j}=O V_{i j}-A V_{i j}
$$

\section{Paso 6. Cálculo de la cantidad de recursos financieros requeridos para comprar la materia prima}

Una vez aplicada la ecuación 13 en la primera operación (última en aplicar esta ecuación), se obtendrá la cantidad de recursos financieros requeridos para comprar la materia prima. Este número será comparado con el resultado obtenido de la aplicación de la ecuación (14) lo cual permitirá conocer si habrá ahorro $\left(I V_{l j}<E R B_{j}\right)$ o pérdida $\left(I V_{l j}>E R B_{j}\right)$ en la compra de la materia prima.

$$
E R B_{j}=\frac{M I_{j} M S T_{j}}{L S B_{j}}
$$

Donde $E R B_{j}$ es el presupuesto necesario estimado para el producto $j, M I_{j}$ es el presupuesto disponible para adquirir el producto $j, M S T_{j}$ es el dinero gastado en el proceso de transporte del producto $j$ y $L S B_{j}$ es tamaño del lote a comprar. Dicho presupuesto estimado será la cantidad de recursos financieros disponible por unidad de materia prima.

\section{RESULTADOS}

En esta sección se mostrarán los resultados del método propuesto. Para esto se tomó como objeto de estudio la cadena de contadores de energía eléctrica (CEE), perteneciente a la Fábrica de Fusibles y Desconectivos (FFD) de la provincia de Villa Clara, Cuba. Esta cadena se dedica a la calibración de contadores de energía eléctrica para luego venderlos empresas encargadas de brindar el servicio eléctrico al país. Al mismo tiempo, necesita materia prima de la Empresa ENERGOIMPORT (suministrador), a la cual realiza la solicitud después de pronosticar la demanda futura. Por ende, se desea determinar el valor agregado de cada uno de las operaciones en cada proceso (aprovisionamiento, producción y distribución) de la cadena objeto de estudio.

Paso 1. Definir precio de venta del producto. El proceso seleccionado para el estudio ocurre en el laboratorio de calibración de contadores de energía eléctrica $(127 \mathrm{v}$ y $220 \mathrm{v})$. Ambos productos atraviesan los mismos procesos y operaciones, por lo que se detallará solo el contador de 127v. Este producto tiene un precio de venta $\$ 12,73$, el que es aprobado por el Ministerio de Finanzas y Precios a partir de una propuesta de precio realizada por la FFD.

Paso 2. Identificar las operaciones de cada proceso. En este paso se identificaron las operaciones en cada uno de los procesos de la cadena de suministro objeto de estudio las que se muestran en la Tabla 2. En el 
Tabla 2. Procesos y operaciones de la cadena de contadores de energía eléctrica.

\begin{tabular}{|l|}
\hline \multicolumn{1}{|c|}{ Proceso de aprovisionamiento } \\
\hline $\begin{array}{l}\text { Compra de la materia prima (OP-1). } \\
\text { Contratación del transporte (OP-2). } \\
\text { Revisión de la materia prima (OP-3). } \\
\text { Transporte de la materia prima hasta el almacén de } \\
\text { materia prima (OP-4). }\end{array}$ \\
\hline \multicolumn{1}{|c|}{ Proceso de producción } \\
\hline $\begin{array}{l}\text { Aprovisionamiento del laboratorio (OP-5). } \\
\text { Preparación de la materia prima (OP-6). } \\
\text { Calibración de los contadores de energía eléctrica } \\
\text { (OP-7). } \\
\text { Almacenaje de productos terminados (OP-8). }\end{array}$ \\
\hline \multicolumn{1}{|c|}{ Proceso de distribución } \\
\hline $\begin{array}{l}\text { Comunicación a los clientes sobre productos terminados } \\
\text { (OP-9). } \\
\text { Servicio postventa (OP-10). }\end{array}$ \\
\hline
\end{tabular}

presente estudio se tomó como punto de referencia la cantidad de unidades compradas al suministrador en enero del 2015, las cuales fueron un total de 400.000 contadores, de estos 300.000 son de $127 \mathrm{v}$. En la Tabla 3 se muestran las capacidades de cada uno de las operaciones calculadas empleando las ecuaciones (1) y (2).

Paso 3. Identificar los requerimientos operacionales. En la cadena objeto de estudio se detectaron como requerimientos operacionales la energía eléctrica, los contadores de energía eléctrica, recursos humanos, sellos, etiquetas (informan lote, fecha de fabricación y de vencimiento y código del

Tabla 3. Capacidad de cada operación de la cadena de suministro $(\mathrm{CEE} / \mathrm{h})$.

\begin{tabular}{|c|c|}
\hline Operaciones & Capacidad \\
\hline OP-1 & 15,552 \\
\hline OP-2 & 66,667 \\
\hline OP-3 & 800,000 \\
\hline OP-4 & 200,000 \\
\hline OP-5 & 625 \\
\hline OP-6 & 500 \\
\hline OP-7 & 225 \\
\hline OP-8 & 500 \\
\hline OP-9 & 186,046 \\
\hline OP-10 & 2,350 \\
\hline
\end{tabular}

operario que la puso) y la información necesaria para maniobrar el producto y los instrumentos.

Paso 4. Cálculo del coeficiente de valor agregado. Los tiempos de procesamiento (en segundos) se calcularon de acuerdo con la ecuación (5) ya que las operaciones clasifican como mecánico manual. El tiempo de retraso (en segundos) fue calculado por la ecuación (7) y el costo (en pesos por unidad) mediante la (8). Cada uno de estos componentes se muestran en la Tabla 4, donde además se observa el peso específico o coeficiente de valor agregado (en por ciento) después de aplicar la ecuación (10).

Tabla 4. Datos para el cálculo del coeficiente de valor agregado de cada operación (\%).

\begin{tabular}{|c|r|r|c|r|}
\hline Ops. & \multicolumn{1}{|c|}{$\boldsymbol{T}_{\boldsymbol{i}}$} & $\boldsymbol{D t}_{\boldsymbol{i} \boldsymbol{j}}$ & $\boldsymbol{C}_{\boldsymbol{i} \boldsymbol{j}}$ & $\boldsymbol{S}_{\boldsymbol{i} \boldsymbol{i}}$ \\
\hline OP-1 & 6,4800 & 6,4755 & 0,0153 & 9,19 \\
\hline OP-2 & 1,0800 & 1,0755 & 0,0129 & 1,80 \\
\hline OP-3 & 0,0045 & 0 & 0,0003 & 0,01 \\
\hline OP-4 & 0,0180 & 0,0135 & 0,0001 & 0,02 \\
\hline OP-5 & 5,7600 & 5,7420 & 0,1745 & 12,33 \\
\hline OP-6 & 7,2000 & 7,1810 & 0,3624 & 19,15 \\
\hline OP-7 & 21,4286 & 21,4096 & 0,3124 & 37,17 \\
\hline OP-8 & 5,6000 & 5,5810 & 0,3895 & 17,67 \\
\hline OP-9 & 0,0190 & 0,0000 & 0,0087 & 0,25 \\
\hline OP-10 & 1,5300 & 1,5110 & 0,0128 & 2,41 \\
\hline
\end{tabular}

Paso 5. Cálculo del valor de cada producto en cada operación. Para el cálculo del valor de cada producto en cada operación se comenzó por determinar el valor agregado neto por medio de la ecuación (11). Luego se aplicó la ecuación (12) para calcular el valor agregado del producto en cada una de las operaciones de la cadena, mostrándose los resultados en la Tabla 5 dados en pesos por artículo. Luego, se determinó el valor inicial y final de cada operación mediante la ecuación (13) tal y como se muestra en la Tabla 6. La misma fue aplicada de atrás hacia adelante, coincidiendo el valor de salida de la última operación con el precio de venta del producto.

\section{Paso 6. Cálculo de la cantidad de recursos financieros requeridos para comprar la materia prima}

Una vez obtenido cada uno de los valores agregado en cada operación, se calculó la cantidad de recursos 
Tabla 5. Datos para el cálculo del coeficiente de valor agregado de cada operación.

\begin{tabular}{|c|c|c|c|}
\hline Ops. & $\boldsymbol{N A}_{\boldsymbol{i} \boldsymbol{j}}$ & $\boldsymbol{A \boldsymbol { C } _ { \boldsymbol { i j } }}$ & $\boldsymbol{A \boldsymbol { V } _ { \boldsymbol { i } \boldsymbol { j } }}$ \\
\hline OP-1 & 0,0331 & 0,0153 & 0,0484 \\
\hline OP-2 & 0,0065 & 0,0129 & 0,0194 \\
\hline OP-3 & 0,0000 & 0,0003 & 0,0003 \\
\hline OP-4 & 0,0001 & 0,0001 & 0,0002 \\
\hline OP-5 & 0,0444 & 0,1745 & 0,2189 \\
\hline OP-6 & 0,0689 & 0,3624 & 0,4313 \\
\hline OP-7 & 0,1338 & 0,3124 & 0,4462 \\
\hline OP-8 & 0,0636 & 0,3895 & 0,4531 \\
\hline OP-9 & 0,0009 & 0,0087 & 0,0096 \\
\hline OP-10 & 0,0087 & 0,0128 & 0,0215 \\
\hline
\end{tabular}

financieros requeridos para comprar la materia prima (contadores sin calibrar). Para ello se aplicó la ecuación (14) $\left(M I_{j}=4400000, M S T_{j}=3880 \mathrm{y}\right.$ $\left.L S B_{j}=400000\right)$ y se obtuvo como resultado que la cadena objeto de estudio dispone de $\$ 10,9903$ por cada unidad de materia prima que desee comprar. Este valor especifica el precio límite que puede pagar la cadena de suministro para obtener cada unidad de materia prima. Por otro lado, se puede observar que el valor inicial de la primera operación en la Tabla 4 (\$ 11,0852) es mayor que la cantidad de recurso monetario con que dispone la cadena para adquirir cada unidad de materia prima lo que se traduce como una pérdida $\left(I V_{l j}>E R B_{j}\right)$.

Tabla 6. Valor de entrada y salida en cada uno de las operaciones.

\begin{tabular}{|c|c|c|c|}
\hline Ops. & $\boldsymbol{I V}_{\boldsymbol{i} \boldsymbol{j}}$ & $\boldsymbol{A} \boldsymbol{V}_{\boldsymbol{i j}}$ & $\boldsymbol{O} \boldsymbol{V}_{\boldsymbol{i j}}$ \\
\hline OP-1 & 11,0852 & 0,0484 & 11,1336 \\
\hline OP-2 & 11,1336 & 0,0194 & 11,1530 \\
\hline OP-3 & 11,1530 & 0,0003 & 11,1533 \\
\hline OP-4 & 11,1533 & 0,0002 & 11,1535 \\
\hline OP-5 & 11,1535 & 0,2189 & 11,3724 \\
\hline OP-6 & 11,3724 & 0,4313 & 11,8037 \\
\hline OP-7 & 11,8037 & 0,4462 & 12,2499 \\
\hline OP-8 & 12,2499 & 0,4531 & 12,7030 \\
\hline OP-9 & 12,7030 & 0,0096 & 12,7126 \\
\hline OP-10 & 12,7126 & 0,0215 & 12,7341 \\
\hline
\end{tabular}

Al mismo tiempo, la diferencia entre los dos valores anteriormente expuestos (pérdida), puede traducirse en un pago de $\$ 3798$ por encima del presupuesto disponible para adquirir la materia prima necesaria
(400.000 unidades), o dejar de comprar 312 contadores sin calibrar (equivalen a 312 productos terminados) e incumplir con la demanda. En el caso de la cadena de suministro objeto de estudio el presupuesto es aprobado a inicio de año, por lo que dicha diferencia constituye una pérdida. Por lo tanto, la única opción de la cadena de suministro para cumplir con la demanda sin presentar pérdidas económicas es reducir $\$ 0,0944$ por cada unidad procesada (en los costos o en la ganancia neta), lográndose de esta forma un presupuesto unitario al inicio de la cadena de $\$ 11,0947$ (superior a $\$ 11,0852$ ).

\section{Discusión de los resultados}

En la sección anterior se aplicó el procedimiento propuesto donde se determinó el valor agregado en cada una de las operaciones, donde la operación que mayor valor agrega es la de almacenaje de productos (envase, embalaje, etc.), seguido por la operación de calibración de los contadores de energía eléctrica, aprovisionamiento del laboratorio, preparación de la materia prima y revisión de la materia prima. Para ello fueron analizados los tiempos de procesamientos, costos y tiempo de retraso. A su vez, el análisis del tiempo de retraso puede ser de gran utilidad en el cálculo de los niveles de inventario (ecuación (15)), constituyendo de esta forma un punto de partida en la identificación y análisis de las operaciones de capacitad limitada.

$$
\begin{gathered}
I L_{i j}(T)=\frac{\left(t_{i j} t_{i 1, j}\right) * V p_{j}(T)}{t_{i j}} \\
\forall\left(t_{i j} t_{i 1, j}\right) \geq 0
\end{gathered}
$$

Donde $I L_{i j}$ es el nivel de inventario de productos $j$ en la operación $i$ en un período de tiempo $T, V p_{j}(T)$ es el volumen de producción del producto $j$ en un período de tiempo $T, L p_{j}$ es el volumen de los lotes de producción y $t t_{i-1, i}$ es el tiempo de transporte del lote de producción de la operación $i-1$ a la $i$.

Por otro lado, la operación de mayor peso o la de mayor valor agregado neto (calibración de los contadores de energía eléctrica) es considerada el cuello de botella, la que no necesariamente tiene que ser la operación de mayor valor agregado. Al mismo tiempo, estos pesos constituyen un punto de partida a la hora de tomar acciones correctivas en estas operaciones. 
A su vez, el valor agregado calculado cambiará cada vez que se realice un aprovisionamiento a la cadena. Esto se debe a que el tiempo de procesamiento y costo en cada operación del proceso de aprovisionamiento varía según la cantidad de artículos a comprar a los proveedores y el número de veces que se necesite abastecer la cadena. Cada aprovisionamiento a la cadena se traduce como variaciones en el costo y en el tiempo de procesamiento de algunas operaciones. Al mismo tiempo, dicho valor agregado se verá afectado al cambiar el costo o el tiempo de procesamiento. Para una mejor comprensión de lo anteriormente abordado, se calculó el valor agregado según un aprovisionamiento de 300.000, 350.000, $400.000,450.000$ y 500.000 unidades. Luego, estos valores agregados fueron llevados a porcientos que representaba del valor agregado total los que se ilustran en la Figura 2.

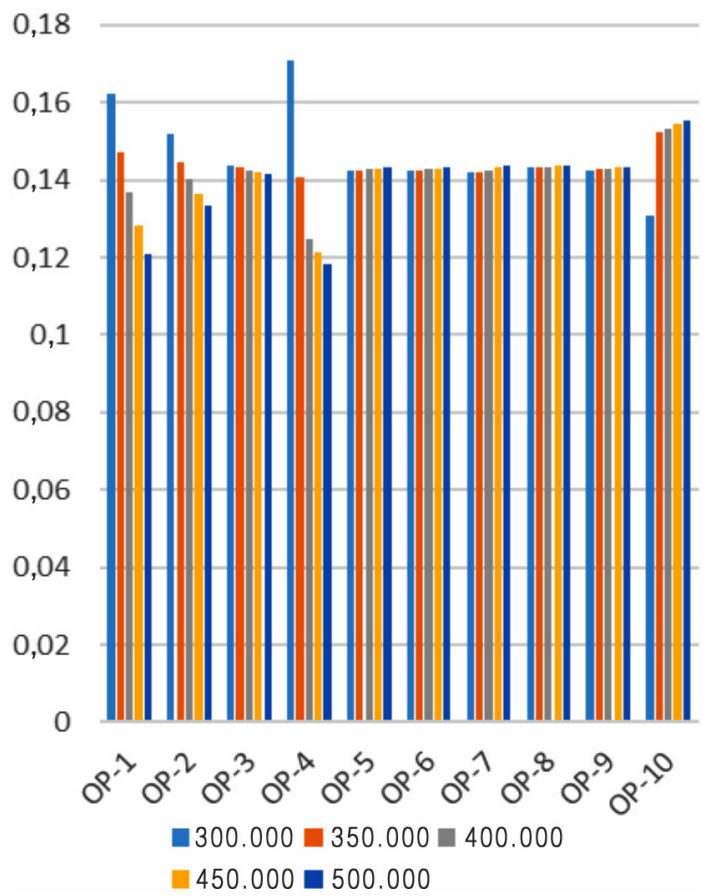

Figura 2. Por cientos de valor agregado en cada operación según su aprovisionamiento.

Como se puede observar en la Figura 2 las operaciones del eslabón aprovisionamiento tienden a disminuir a medida que se aumenta la cantidad de materia prima. Al mismo tiempo, el aumento de este aprovisionamiento a la cadena aumenta el valor agregado en las operaciones pertenecientes al eslabón de producción y distribución respectivamente. Tal variación se debe a la disminución de los costos indirectos con el aumento de la cantidad de artículos a procesar.

\section{CONCLUSIONES}

El método presentado, a diferencia de los demás identificados en la literatura consultada, permite calcular el valor agregado en cada una de las operaciones de la cadena objeto de estudio. Además, puede ser aplicada a procesos específicos de la cadena de suministro o combinación de estos. Al mismo tiempo, brinda información vital para la planificación de la cadena en cuanto a la importancia de las operaciones.

También, ofrece la posibilidad de considerar el tiempo de retraso para el cálculo del valor agregado en cada una de las operaciones de la cadena objeto de estudio, el que no ha sido considerado en ninguna de las investigaciones consultadas. Conjuntamente, dicho tiempo de retraso puede ser empleado para el cálculo de los niveles de inventario, como punto de partida en la identificación y análisis de las operaciones de capacidad limitada.

Por otro lado, el cálculo del valor agregado según el método presentado en este trabajo engloba de manera más completa la información en cada una de las operaciones de los procesos de la cadena de suministro. Al mismo tiempo, este puede ser aplicado a diversos procesos de producción de bienes o servicios considerando en todos ellos, cada una de las operaciones que intervienen en los procesos de aprovisionamiento, producción y distribución. Además, permite conocer los valores iniciales y finales de cada una de las operaciones, conociendo de esta forma la existencia de posibles ahorros o pérdidas en el proceso de compra de la materia prima.

Sin embargo, el método propuesto tiene como limitante que solo analiza componentes económicos para determinar el valor agregado. En tal sentido, si se añadieran componentes sociales y ambientales, se obtendría una valoración desde el punto de vista sostenible de la cadena de suministro, brindando de esta forma el valor que aporta cada una de las operaciones de la cadena a la sostenibilidad. 


\section{AGRADECIMIENTOS}

Agradecemos a la empresa de producciones electromecánicas por las facilidades brindadas para el desarrollo de la presente investigación.

\section{REFERENCIAS}

[1] F. Dweiri and S. Khan. "Development of a universal supply chain management performance index". International Journal of Business Performance and Supply Chain Modelling. Vol. 4 No 3/4, pp. 232-236. 2012.

[2] S. George and S. Mathew. "Improving financial performance of supply chain in a spice industry". International Journal of Science Technology \& Engineering. Vol. 1 $\mathrm{N}^{\circ}$ 3, pp. 14-20. 2014

[3] F. Costantino, G. Di Gravio, A. Shaban and M. Tronci. "A real-time SPC inventory replenishment system to improve supply chain performances". Expert Systems with Applications. Vol. $42 \mathrm{~N}^{\circ} 3$, pp. 1665-1683. 2015.

[4] T. Sawik. "On the fair optimization of cost and customer service level in a supply chain under disruption risks". Omega. Vol. 53 No 2015, pp. 58-66. 2015.

[5] M. Christopher. "Logistics and supply chain management: Strategies for reducing cost and improving service". Prentice Hall Financial Times. 2da edición. Londres, Inglaterra. 1998.

[6] R. Rajnoha, A. Sujová and J. Dobrovič. "Management and Economics of Business Processes Added Value". Procedia-Social and Behavioral Sciences. Vol. $62 \mathrm{~N}^{\mathrm{o}} 2012$, pp. 1292-1296. 2012.

[7] M. Porter and V. Millar "How information gives you competitive advantage". Harvard Business Review. Vol. $63 \mathrm{~N}^{\mathrm{o}} 4$, pp. 149-155.1985.

[8] J. Katz and M. Boland. "A new value-added strategy for the US beef industry: the case of US Premium Beef Ltd". Supply Chain Management: An International Journal. Vol. $5 \mathrm{~N}^{\circ}$ 2, pp. 99-110. 2000.

[9] L. Horvath. "Collaboration: the key to value creation in supply chain management". Supply Chain Management: An International Journal. Vol. $6 \mathrm{~N}^{\circ}$ 5, pp. 205-207. 2001.

[10] H. Dekker. "Value chain analysis in interfirm relationships: a field study". Management
Accounting Research. Vol. 14. $\mathrm{N}^{\mathrm{o}} 1$, pp. 1-23. 2003.

[11] A. Vinajera, F. Marrero and W. Sarache. "Procedure for calculating the value added. An application in the medium-voltage fuses industry". Revista Ingeniería e Investigación. Vol. $34 \mathrm{~N}^{\mathrm{o}}$ 1, pp. 90-94. 2014.

[12] A. Acevedo-Tirado, M. Ruiz-Morales and O. Lobato-Calleros. "Additional Indicators to Promote Social Sustainability within Government Programs: Equity and Efficiency". Sustainability Vol. $7 \mathrm{~N}^{\mathrm{o}} 7$, pp. 9251-9267. 2015.

[13] S. Khan, D. Chouhan, D. Candra and D. Ssubhamgoswani. "Measurement of Value Creation Vis-À-Vis EVA: Analysis of Select BSE Companies". Pacific Business Review International. Vol. $5 \mathrm{~N}^{\circ}$ 3, pp. 114-132. 2012.

[14] G. Michalski G. "Portfolio management approach in trade credit decision making". Romanian Journal of Economic Forecasting. Vol. $3 \mathrm{~N}^{\mathrm{o}}$ 2007, pp. 42-53. 2007.

[15] A. Fearne, M. García Martínez and B. Dent B. "Dimensions of Sustainable Value Chains: Implications for Value Chain Analysis". Supply Chain Management: An International Journal. Vol. 17 No 6, pp. 575-581. 2012.

[16] M. Largani, M Kaviani and A. Abdollahpour. "A review of the application of the concept of Shareholder Value Added (SVA) in financial decisions". Procedia-Social and Behavioral Sciences. Vol. 40 No 2012, pp. 490-497. 2012.

[17] C. Soosay, A. Fearne and B. Dent B. "Sustainable Value Chain Analysis - A Case Study Of Oxford Landing From 'Vine to Dine".. Supply Chain Management: An International Journal. Vol. $1 \mathrm{~N}^{\circ} 1$, pp. 68-77. 2012.

[18] C. Koedel, K. Mihaly and J. Rockoff. "Valueadded modeling: A review". Economics of Education Review. Vol. 47, pp. 180-195. Agosto 2015. 2015.

[19] J. Wang. "Encyclopedia of Business Analytics and Optimization". IGI Global. Hershey, USA., pp. 1457-1478. 2014.

[20] R. Sathre and L. Gustavsson. "Process-based analysis of added value in forest product industries". Forest Policy and Economics. Vol. $11 \mathrm{~N}^{\circ}$ 1, pp. 65-75. 2009.

[21] J. Stern, G. Stewart and D. Chew. "The EVA financial management system". Journal of 
Applied Corporate Finance. Vol. $8 \mathrm{~N}^{\mathrm{o}} 2$, pp. 32-46. 1995.

[22] Z. Xin'e, W. Ting and Z. Yuan. "Economic Value Added for Performance Evaluation: A Financial Engineering"' Systems Engineering Procedia. Vol. 5 No 2012, pp. 379-387. 2012.

[23] A. Fernandes. "Assessing the technology contribution to value added". Technological Forecasting and Social Change. Vol. $79 \mathrm{~N}^{\circ} 2$, pp. 281-297. 2012.

[24] P. Longinidis and M. Georgiadis."Managing the trade-offs between financial performance and credit solvency in the optimal design of supply chain networks under economic uncertainty". Computers \& Chemical Engineering. Vol. 48 No 2013, pp. 264-279. 2013.

[25] V. Burešová and L. Dvořáková. "Possibilities and Difficulties of Economic Value Added Use 2 Problem Formulation". Recent Researches in Business Administration, Product Design and Marketing: Proceedings of the 4th International Conference on Design and Product Development (ICDPD'13), Proceedings of the 4th International Conference on Business Administration (ICBA'13). Chania, Crete Island, Greece. 2013.

[26] M. Woods, L. Taylor and G. Fang. "Electronics: A case study of economic value added in target costing". Management Accounting Research. Vol. 23 No 2012, pp. 261-277. 2012.

[27] R. Schonberger. "Measurement of Lean Value Chains: Efficiency and Effectiveness". Modelling Value, pp. 65-75. Springer. Washington, USA. 2012.

[28] M. Christopher. "Logistics and supply chain management: creating value-added networks". Prentice Hall Financial Times. Cuarta edición. Grean Bretain. 2005.

[29] P. Fernández. "Shareholder Value Creation: A Definition". Social Science Research Network, pp. 1-10. November, 2015.

[30] J. Bacidore, J. Boquist, T. Milbourn and A. Thakor. "The search for the best financial performance measure". Financial Analysts Journal. Vol. 52 No 3, pp. 11-20. 1997.

[31] F. Figge and T. Hahn. "Sustainable value added: A New Approach to Measuring Corporate Sustainable Performance". Managing the Business Case for Sustainability: The
Integration of Social, Environmental and Economic Performance. Vol. $146 \mathrm{~N}^{\circ} 164$, pp. 146-164. 2002.

[32] P. Fernández. "EVA, Economic profit and cash value added do NOT measure shareholder value creation". Fecha de publicación: 22 de mayo de 2001. Fecha de consulta: 11 febrero de 2015. URL: http://citeseerx.ist. psu.edu/viewdoc/download?doi=10.1.1.20 $1.9225 \&$ rep $=$ rep $1 \&$ type $=$ pdf

[33] H. Nakhaei and N. Hamid. "Is refined economic value added more associated with stock return than accounting measures? The Malaysian evidence". Society and Economy in Central and Eastern Europe. Vol. $38 \mathrm{~N}^{\circ} 1$, pp. 69-85. 2016.

[34] M. Pourali and Z. Roze. "The Relationship between Market Value Added with Refined Economic Value Added and Performance Accounting Criteria in the Firms listed in the Tehran Stock Exchange". International Research Journal of Applied and Basic Science. Vol. 4 No 6, pp. 1636-1645. 2013.

[35] T. Fletcher y N. Russell-Jones. "Política de precios: cómo crear el máximo valor a través de una fijación de precios efectiva". Gestión 2000, pp. 478. Barcelona, España. 2002.

[36] T. Nagle y R. Holden. "Estrategia y tácticas para la fijación de precios: Guía para tomar decisiones beneficiosas". Granica S.A. Buenos Aires, Argentina, pp. 573. 1995.

[37] E. Aghezzaf. "Lot-sizing problem with setup times in labor-based capacity production systems". International Journal of Production Economics. Vol. $64 \mathrm{~N}^{\mathrm{o}}$ 1-3, pp. 1-9. 2000.

[38] F. Marrero-Delgado. "Procedimientos para la toma de decisiones logísticas con enfoque multicriterio en la cadena de corte, alza y transporte de la caña de azúcar. Aplicaciones en el CAI de la provincia de Villa Clara". Tesis para optar al grado de doctor en ciencias técnicas. Departamento de Ingeniería Industrial. Universidad Central "Marta Abreu" de Las Villas. 2001.

[39] F. Figge and T. Hahn. "Sustainable Value Added-measuring corporate contributions to sustainability beyond eco-efficiency". Ecological Economics. Vol. 48, pp. 173187. 2004. 